\title{
"Defense" injuries in attacks on humans by domestic dog (Canis lupus familiaris) and jaguar (Panthera onca)
}

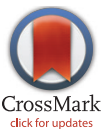

\author{
Manoel Francisco de Campos Neto', Vidal Haddad Junior ${ }^{2 *}$ \\ 'The Forensic Medicine Section, POLITEC -Cáceres, Mato Grosso State, Brazil \\ ${ }^{2}$ Department of Dermatology, Botucatu Medical School, São Paulo State University, São Paulo, Brazil
}

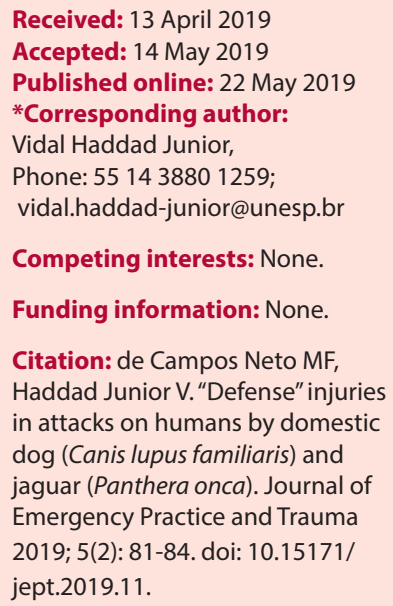

\begin{abstract}
This communication describes two attacks by domestic and wild carnivores in Caceres County, localized in the Pantanal area, an extensive flooded plain in Mato Grosso State, Midwest region of Brazil. The first attack took place in an urban area and was caused by a Rottweiler dog (Canis lupus familiaris) created by the family of the victim. Another attack occurred in a rural area, caused by a jaguar (Panthera onca), which was disturbed while feeding, characterizing an unprovoked attack. Both the patients were wounded in the forearm, with different severity of the wounds. The lesions restricted to the forearm were the consequence of the victims using their arms as a shield, featuring the "defense" injuries, but the severity of the wounds were proportional to the strength of the attacking carnivore. Health staffs and emergency centers should be alert for soft tissue and bone injuries in the observed body regions after domestic and wild animal attacks.

Keywords: Wildlife, Domestic animals, Animal bites, Attacks on humans
\end{abstract}

\section{Introduction}

Animal bites account for $1 \%$ of emergency room attendance in the USA, caused mainly for domestic animals (1). Dogs (Canis lupus familiaris) are the animals that cause most of the injuries ( $80 \%$ to $90 \%$ ). The blunt teeth of dogs and the strength of their jaws cause lacerations, cuts and crushing of tissues, which result in lesions of deep structures such as muscles, vessels, tendons and bones (2-4).

Wildlife may also be associated with human injuries, which can be serious depending on the animal and circumstances of attack. The largest cat in the Americas and the third largest in the world is the jaguar (Panthera onca). These animals can measure five to six feet from the nose to the tip of the tail and weigh 140 to 220 pounds (5). The formidable canine teeth of the jaguar are used to kill prey and tear the flesh. Jaguars kill prey by piercing their brains through skull bites. Other teeth, such as incisors, are flattened and scrape small bits of meat from the bones of prey $(4,6)$. In some parts of South America, it is still possible to find jaguars with relative facility and these meetings are more likely to happen to local residents and tourists $(6-8)$.

Bacterial secondary infections are common after bites of dogs and big cats. The species of bacteria associated with dogs bites are Staphylococcus sp., Streptococcus sp., Eikenella corrodens, Pasteurella multocida, Proteus sp., Klebsiella sp. and others. The bites of jaguar predispose infections mainly by streptococci and staphylococci and rarely by Pasteurella multocida $(7,8)$.

This case report describes two attacks by domestic and wild carnivores in Caceres County, localized in Mato Grosso State, Midwest region of Brazil. The first attack took place in an urban area and was caused by a Rottweiler dog created by the family of the victim. These dogs can reach $60 \mathrm{~kg}$ and have a bite in "scissor" in which the superior and inferior incisors teeth touch. The force of the bite can reach almost $150 \mathrm{~kg}$. The other attack occurred in a rural area, caused by a jaguar in the Pantanal area, an extensive flooded plain in the states of Mato Grosso and Mato Grosso do Sul, Brazil. Jaguars have extremely powerful bites that can reach $270 \mathrm{~kg}$. The circumstances and clinical aspects of the attacks as well as injuries to victims are discussed.

\section{Cases Reports}

Case 1 - (Rottweiler Dog)

A 9-year-old male attended Cáceres Regional Hospital (Mato Grosso State, Brazil) in October 2014. The patient 
was brought by his father, who reported an attack by the rottweiler breed dog of the family. The bites occurred when the child gave food to the animal, which he usually did not. The victim tried to defend himself by putting his arm in front of body and face.

On physical examination, the patient complained of severe pain in the left arm. He could not extend the 3rd and 4 th fingers and had numbness in a part of the hand. The $1 / 3$ medium/lower forearm showed extensive and deep lacerations/perforations, blunt injuries in edges with extrusion of muscle fibers ("pullout" injuries) and complete section of extensor tendons (Figure 1). The wounds were contaminated with grains of sand and earth and bled profusely due to ruptured muscular branches of arteries. The simple radiological examination showed severe tissue destruction and no fractures.

The patient was taken to the operating room and extensive washing was done with saline solution $9 \%$, antisepsis with topic povidone and debridement with excision of devitalized tissues. Hemostasis was performed with simple catgut 3-0, the repair of ruptured tendons utilized mononylon 3-0 and the exploitation of the radial nerve showed its total section. The surgical correction used rotation skin flaps seeking coverage of injuries, placement of Penrose drains to avoid blood collections in the immediate postoperative period and muscular, subcutaneous and skin sutures with mononylon 4-0 and compressive dressings. The patient was also treated with intravenous hydration with glucose-saline solution, maintenance of venous access and cephalothin $1.0 \mathrm{~g} \mathrm{IV}$ 6/6 hours; gentamicin $60 \mathrm{mg}$ IV 8/8 hours; dipyrone 1.5 $\mathrm{ml} \mathrm{EV} \mathrm{6/6} \mathrm{hours;} \mathrm{diclofenac} 50 \mathrm{mg}$ PO 8/8 hours; SAT (anti-tetanus serum $5000 \mathrm{U}$ IM) and daily dressings with mobilization of drains. The rabies vaccination was not carried out. About a month later, the child had extensive scarring at the site of bites and limitation of the extension of movements of the 3rd and 4th left fingers and areas of decreased sensitivity of the regions innervated by the sensory branch of the left radial nerve.

\section{Case 2 - Jaguar}

A 46-year-old male attended Cáceres Regional Hospital (Mato Grosso State, Brazil). Cáceres is a municipality located in the Pantanal region, a lowland flooded area featuring intense wildlife activity.

The victim reported that he was attacked by a jaguar (Panthera onca), when he was in a pasture with other workers looking for a missing horse. He felt a strong smell and imagined that it could be of the dead horse and found an alligator carcass. The jaguar that was devouring the carcass appeared and attacked the patient, who tried to defend himself by raising his arm in front of his face and was bitten. The attack caused lacerated skin, muscle lesions and open fractures of the bones of the left forearm (Figure 2). The dogs who accompanied the patient

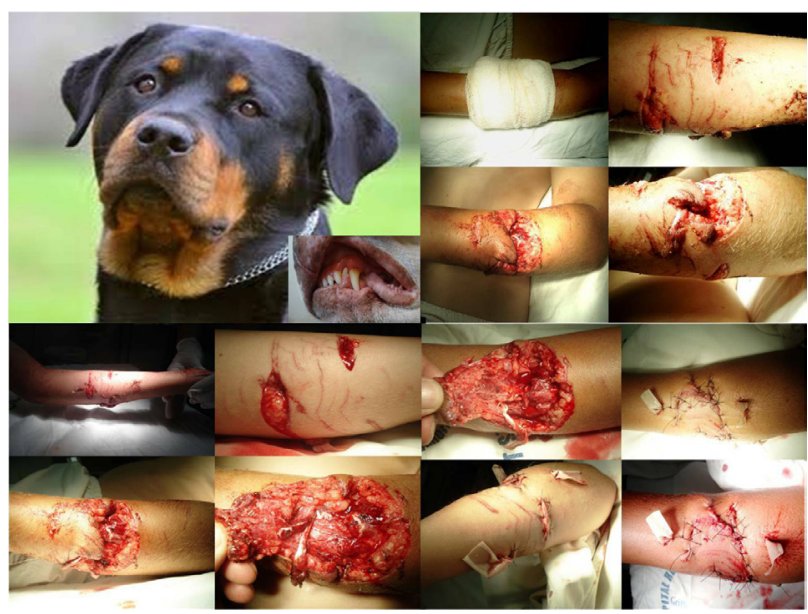

Figure 1. Rottweiler dog and its teeth in detail. The 1/3 medium/ lower forearm shows extensive and deep lacerations/perforations, blunt injuries in edges with extrusion of muscle fibers and section of extensor tendons. The sequence shows the cleaning of the wound and the surgical treatment.

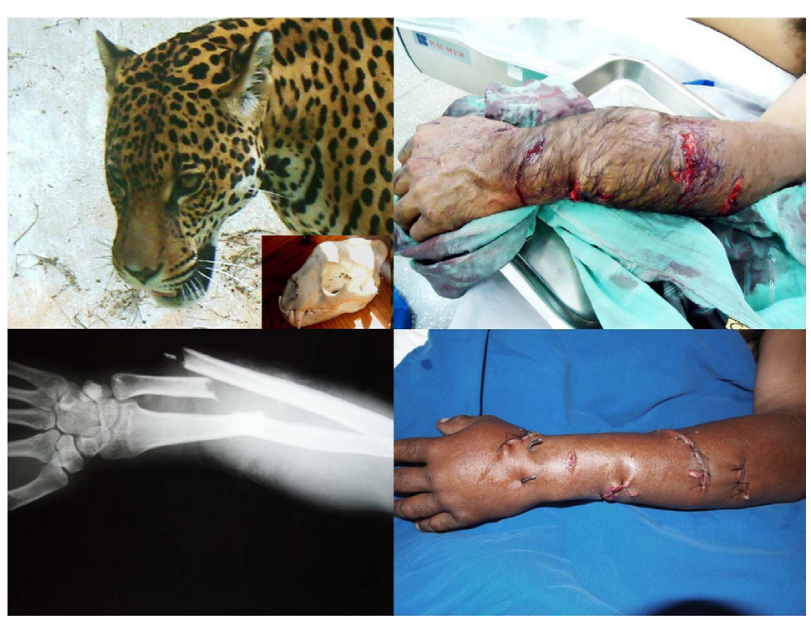

Figure 2. Jaguar (Panthera onca) and the skull showing the teeth. Note the lacerated skin, muscle lesions and open fractures of the bones of the left forearm of the victim and the surgical treatment described in the text.

attacked the jaguar, causing it to deflect the attack and leave the victim. When dogs reacted, the animal climbed on a tree and soon after, tried to attack the victim again, but it was driven away by the fellows who arrived on the scene.

The patient was referred to the Cáceres Regional Hospital and taken into the emergency room. The exam showed extensive lacerations on his left forearm, with open fractures next to the wrist. Simple radiological examinations revealed radius and ulna fractures, with axis deviation and presence of fragments in the area. The injuries were washed thoroughly with saline solutionand antisepsis was performed with povidone. The devitalized tissues were extracted and after orthopedic surgery, the fractured bones were aligned and fixed with rods. 
The treatment also utilized Penrose drains, sutures by planes with mononylon 4-0 and compressive dressings. Prevention of infection was performed with Cephalothin $1.0 \mathrm{~g} \mathrm{IV} \mathrm{6/6} \mathrm{hours} \mathrm{and} \mathrm{gentamicin} 60 \mathrm{mg}$ IV 8/8 hours. The SAT (anti-tetanus serum) was applied at a dose of 5000 U IM. He was vaccinated against rabies (05 doses intramuscularly), received rabies immunoglobulin (20 $\mathrm{IU} / \mathrm{kg}$ ) and daily dressings. The patient progressed satisfactorily and was discharged after six months of follow-up.

\section{Discussion}

The animals that caused the injuries are carnivores and they present different teeth and mechanical effects of the bites. The resulting injuries, however, happened in the same body segments of the victims and showed morphological similarity.

The treatment of extensive injuries by carnivores involves first aid measures and late care for the treatment of secondary infections and repair of the damaged tissue. The patient should be handled with gloves due to the risk of infections and the presence of blood in the wound. If there is no severe bleeding, the wound should be washed with running water (if possible with pressure) and soap for 5 to 10 minutes, withdrawing all foreign bodies. The bleeding can be dealt with by compressing the wound with a clean cloth. Careful cleaning is more decisive in preventing infections than prophylactic use of antibiotics $(3,4,8)$.

Devitalized tissue should be removed, especially in the wound edges, which occurs very frequently in the dog bites due to the crushing of the tissues. Late injuries, wounds localized in the hands and non-disfiguring wounds can remain open, but should be re-evaluated in 48-72 hours after the initial consultation to detect complications. Large wounds should be sutured with due care $(3,8)$.

The use of antibiotics is necessary if there is the appearance of signs of local infection at the wound such as edema, erythema and purulent discharge. The antibiotic of choice in such situations is the amoxicilina/clavulanic acid, $500 \mathrm{mg}$ PO 6/6 hours for ten days. Other options are penicillin and cephalosporins of the second and third generation $(2,7,8)$. Patients without tetanus immunization should receive the vaccine and tetanus toxoid. In patients immunized for rabies without reinforcement for over five years, is required application of the tetanus toxoid. The rabies must be prevented in any wild animal bite, especially carnivores. Patients bitten by wild or unknown animals must be vaccinated against rabies (05 doses intramuscularly) and receive rabies immunoglobulin (20 $\mathrm{IU} / \mathrm{kg}$ ). The infiltration of a party on site is useful and the remaining doses shall be applied intramuscularly $(2,3,8)$. The patients presented extensive skin lacerations and fractures. The cases had a high risk of infections, once there were deep punctures, lacerated/crushed tissue and fracture contamination. The antibiotics were used according to the recommendations for carnivores bites) $(1,3,4,8)$. The tetanus prophylaxis was applied and the rabies post-exposure prophylaxis treatment were done for the patient wounded by the jaguar.

\section{Conclusions}

The violence of the jaguar's bite was associated with the double fracture of the victim's forearm bones, which did not occur in Rottweiler dog bites, whose teeth are more likely to crush tissues and rarely cause bone injuries. In both attacks, however, it is clear that injuries were associated with defense attempts by the victims, with their arms brought forward for face and body protection. This movement causes higher exposition to more serious injuries, even in attacks of less impact and not intended to predation, as observed in the cases described.

Attacks by jaguars can be provoked or non provoked (very rare). This attack was considered provoked, due to the fact that females with cubs, animals feeding or hunting ground invasion are stimuli that make the feline attack.

The dog attack was on a child who did not feed it. This can be the cause of aggression. These attacks without the intention of predation are movements that rarely lead to death, intending to scare the victim. The injuries restricted to the forearm are the consequence of the victims using their arms as a shield, featuring the "defense" injuries in the victims. Health staff and emergency centers should be alert for soft tissue and bone injuries after domestic and wild animal attacks.

\section{Ethical issues}

The Cáceres Regional Hospital did not seek for ethical authorization in reporting the cases.

\section{Authors contributions}

Authors declare that the attendance of the cases and the preparation of the manuscript were divided among them. Authors worked collaboratively and take the responsibility on the content and images of this work.

\section{References}

1. Bradford JE, Freer L. Bites and injuries inflicted by wild and domestic animals. In: Auerbach P, ed. Wilderness medicine. 6th ed. St Louis, MO: Mosby; 2012.

2. Haddad V Jr, Neto MF, Mendes AL. Mordeduras de animais (selvagens e domésticos) e humanas. Rev Patol Trop 2013; 42(1): 13-9. doi: 10.5216/rpt.v42i1.23587.

3. Baxter DN. The deleterious effects of dogs on human health: dog-associated injuries. Community Med 1984; 6(1): 29-36.

4. Neto MF, Garrone Neto D, Haddad V Jr. Attacks by jaguars (Panthera onca) on humans in central Brazil: report of three cases, with observation of a death. Wilderness Environ Med 2011; 22(2): 130-5. doi: 10.1016/j.wem.2011.01.007. 
5. Michalski F, Boulhosa RLP, Faria A, Peres CA. Humanwildlife conflicts in a fragmented Amazonian forest landscape: determinants of large felid depredation on livestock. Anim Conserv 2006; 9(2): 179-88. doi: 10.1111/j.1469-1795.2006.00025.x.

6. Silver SC, Ostro LET, Marsh LK, Maffei L, Noss AJ, Kelly MJ, et al. The use of camera traps for estimating jaguar Panthera onca abundance and density using capture/ recapture analysis. Oryx 2004; 38(2): 148-54. doi: 10.1017/ S0030605304000286.

7. Burdge DR, Scheifele D, Speert DP. Serious Pasteurella multocida infections from lion and tiger bites. JAMA 1985; 253(22): 3296-7.

8. Goldstein EJ. Management of human and animal bite wounds. J Am Acad Dermatol 1989; 21(6): 1275-9. 